\title{
Self-sustained oscillator as a model for explosion quakes at Stromboli Volcano
}

\author{
S. De Martino ${ }^{1,3}$, C. Godano ${ }^{2}$, and M. Falanga ${ }^{1,3}$ \\ ${ }^{1}$ Department of Physics, Salerno University, Via S. Allende, Baronissi (SA) 84084 Italy \\ ${ }^{2}$ Dipartimento di Scienze Ambientali, II Universita' di Napoli, Caserta, Italy \\ ${ }^{3}$ INFM unita' di Salerno, 84081 Baronissi (SA), Italy
}

Received: 8 March 2001 - Revised: 1 June 2001 - Accepted: 25 June 2001

\begin{abstract}
We analyze seismic signals produced by explosion-quakes at Stromboli Volcano. We use standard nonlinear procedures to search a low-order effective dynamics. The dimension of the reconstructed phase space depends on the number of samples. Namely larger time lengths correspond to dynamical systems of different complexity. If we restrict the analysis to the signal associated directly to the source (Chouet et al., 1997), we obtain a phase space dimension equal to two. We reproduce this part of the signal with a simple single self-sustained oscillator.
\end{abstract}

\section{Introduction}

In basaltic eruptions, the relative motion of the gas with respect to the liquid produces either an anular flow (Hawaian Fire Fountains) or a Slug flow (Strombolian explosions). Both behaviours are generated by complex processes of magma flow and turbulent degassing. The dynamics underlying the generation of these behaviours is not well understood, even though experiments on laboratory scale (Jaupaurt et al., 1988; Mader et al., 1994) have reproduced well some of their characteristics.

Theoretical models have been produced and much insight on the processes has come from acoustic emission studies (Ripepe et al., 1999; Schik et al., 1988; Vergniolle et al., 1996) . In this paper we focus on the Strombolian explosion quakes to reconstruct a dynamical system that can represent the source in this regime. We assume a complementary point of view, compared with the many fruitful models previously quoted that look at the generation of a slug from the degassing process and that want to reproduce the various features of acoustic emission and seismic signals. In fact, following the line of the seminal paper of Chouet and Shaw (Chouet et al., 1991) we want to extract from the explosion quakes signals recorded at Stromboli some "essential dynamics". In other words, we seek a dynamical model that

Correspondence to: S. De Martino (demartino@sa.infn.it) can represent either the average properties or some of the universal features of the complex dynamics generating these seismic signals. We use standard methods (see, for example Abarbanel, 1995, and references therein) to extract essential dynamics from the the experimental time series. As we shall see the phase space dimension of the "effective dynamical system", in our case, depend on the time length of the sample. This is due to the fact that by increasing the sample length we look at the same dynamical system, with a more and more complex structure. We find that the correlation dimension of the attractor (which gives the number of variables involved in the effective dynamics) is in the range $d_{a}=2 \div 3$. Then there are low-dimension dynamics that can be considered as an effective description of a complex physical system that generates the signal. Starting from this result, we try to simulate the first few seconds with a simple self-sustained oscillator. It is actually in this part that conventional wisdom recognizes a well distinguished trace of the source. We obtain the true signal from the analytical model in the regime of a limit cycle.

\section{Data}

We select explosion-quakes to study seismic signals recorded in April 1992 with small arrays of seismometers, by USGS, University of Aquila and Vesuvian Observatory. An accurate description of the deployed network and the instrumentation can be found in Chouet et al. (1997). We are specifically interested in the following: the wavefield effectively comes from craters and it is composed of body waves in the first few seconds (see also Del Pezzo et al., 1992). In order to extrapolate an "effective" dynamics from the scalar time series, we apply some standard techniques of smoothing. The aim of these procedures (described in the following) is to eliminate the high dimensionality due to the scattering with respect to the dimensions of the source dynamics; note that these operations do not affect the spectral content of the signal. First, we introduce the usual instrumental correc- 


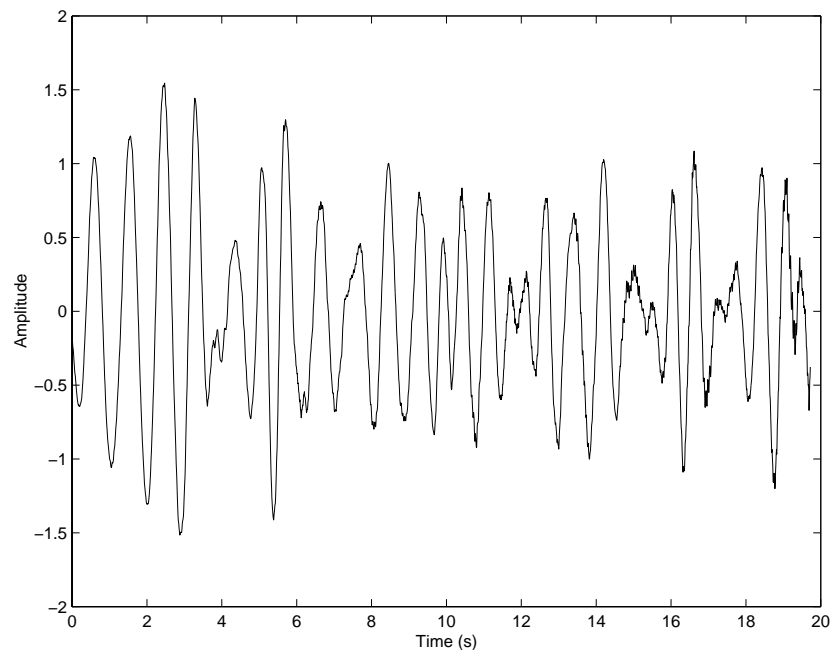

Fig. 1. Normalized amplitude of the average earthquake after the application of the nonlinear denoising.

tion. Then we construct an average earthquake using beam forming based on the knowledge of the apparent velocity of the first pulse and of the position of the stations. This average signal was built using 70 explosion-quakes recorded at 15 stations. This filters the background scattering radiation generated by the random distribution of the points of scattering. Then we correct the signal for the envelope in order to make it stationary at the second order, i.e. we impose that the signal has to mean of zero and constant variance. This is necessary since superimposed dissipative dynamics causes the phase space to contract at a point, preventing the presence of an attractor representative of effective dynamics. Finally, we eliminate the noise by means of a nonlinear technique. We prefer to use this method rather than the standard linear filter. Namely the signals from nonlinear sources can exhibit genuine broad band spectrum and there is no justification to identify any part of spectrum as noise as necessary using spectral or other linear filters. The nonlinear denoising takes into account the fact that deterministic signals form smeared-out lower dimensional manifolds, then the denoising identifies such a structure and projects the signal onto these manifolds in order to reduce the noise (see Grassberger et al., 1993; Kostelich et al., 1993). The final signal is shown in Fig. 1. Again, these transformations do not affect the spectral content of the signal. Now we are ready to perform our analysis.

\section{Phase space reconstruction}

As stated in the introduction, we wish to recognize the low dimension dynamics of the seismic signal recorded at Stromboli Volcano by reconstructing the phase space.

It is well-known that any dynamic process is characterized by the trajectories in the phase space. In some cases they are confined to a limited portion of the whole space defining an attractor of the dynamics. Sometimes the dimension of the attractor is fractal, thus the attractor is called strange. A standard procedure in the analysis of experimental data to reconstruct the asymptotic time evolution is to use the time delay method. This method relays on the mathematical formulation due to Takens (1981). If

$\left\{s_{i}\right\}_{i=1}^{n}$

is a time series of $n$ scalar observations sampled at equal intervals (our explosion-quake signal), the reconstructed attractor consists of points of the form

$x_{i}=\left(s_{i}, s_{i+\tau}, \ldots, s_{i+(m-1) * \tau}\right)$,

where $\mathrm{m}$ is the embedding dimension and $\tau$ is the time delay. Takens shows, under suitable hypotheses, that this reconstruction is equivalent to the original attractor if $m$ is large enough. Thus the numerical problem is to determine $m$ and $\tau$. There is a lot of literature on this problem (see, for example, Abarbanel, 1995). Among different but in essence equivalent methods we select mutual information (Fraser et al., 1986) and false nearest neighbours (Kennel et al., 1992) to calculate, respectively, the time delay and embedding dimension. Mutual information is the extension of the autocorrelation function to the nonlinear domain. The false nearest neighbours technique is based on the projection of the points of the dynamics onto spaces of increasing embedding dimensions. The points appear nearest to the neighbours of some others until the dimension becomes the proper one of our embedding space. When the fraction of these false nearest neighbours with respect to the total number of points is zero, then we can stop our process. Note that the value of $m$, so obtained, does not represent the value of the embedding dimension used to estimate the dimension of the attractor $d_{a}$; indeed it represents the lower limit for the embedding dimension necessary to evaluate $d_{a}$. Moreover, it is an upper bound for $d_{a}$. Now we are able to estimate the dimension of the attractor. In order to do this, we use the standard technique of Grassberger et al. (1983) based on the calculation of the correlation integral

$C(l)=\frac{1}{N} \sum_{i} \frac{1}{N-1} \sum_{j \neq i} \vartheta\left(l-\left|\boldsymbol{x}_{i}-\boldsymbol{x}_{j}\right|\right)$,

where $x_{i}$ are the vectors previously defined in Eq. (2), and $\vartheta$ is the Heaviside function. The slope of $C(l)$ in the scaling region (where it has a power law behaviour) is the correlation dimension of the attractor. Here we evaluate $d_{a}$ first in a moving window of $3.5 \mathrm{~s}$. This choice of window length is due to our capability to model only about $3.5 \mathrm{~s}$ of the signal: we have to compare $d_{a}$ of experimental data with $d_{a}$ of simulated signal. $C(l)$ can be evaluated for different values of the embedding dimension, up to a value at which $d_{a}$ saturates. In Figs. $2 \mathrm{a}-2 \mathrm{~d}$ we show $d_{a}$ versus $m$ for the value of $\tau(0.07 \mathrm{~s}$ for all windows) selected with mutual information. Then we perform the same analysis on the other two windows (10 and $20 \mathrm{~s})$. Although the estimated $d_{a}$ for the last two windows is not completely comparable, due to the different accuracy 
Table 1. The correlation dimensions $d_{a}$ for the adjacent time windows of $3.5 \mathrm{~s} ; m$ is the embedding dimension as evaluated by means of false nearest neighbours

\begin{tabular}{ccc}
\hline Time windows $(3.5 \mathrm{~s})$ & $d_{a}$ & $m$ \\
\hline first window & 1.92 & 2 \\
second window & 1.90 & 2 \\
third window & 1.93 & 3 \\
forth window & 1.94 & 3 \\
\hline
\end{tabular}

Table 2. The correlation dimensions $d_{a}$ for the time windows, respectively, of $10 \mathrm{~s}$ and $20 \mathrm{~s}$. Again $\mathrm{m}$ is the embedding dimension as evaluated by means of false nearest neighbours

\begin{tabular}{ccc}
\hline Time windows (s) & $d_{a}$ & $m$ \\
\hline 10 & 2.4 & 3 \\
20 & 2.8 & 5 \\
\hline
\end{tabular}

of the evaluation for $d_{a}$, these values contain some information on the evolution of dynamics and on the stationarity of our signal. The choice of the window length is also determined from the results of Chouet et al. (1997) who suggest that the source is limited to the first $10 \mathrm{~s}$ of the signal while the other part is affected by strong scattering. The analogue of Fig. 2 for the other two windows is shown in Fig. 3. The dimensions of the various attractors, reproducing the effective dynamics for the three different window lengths, change from about two up to about three (see Tables 1 and 2).

These results are in good agreement with the previous ones: Capuano et al. (1999) obtained a fractal dimension $d_{a}=2.75$ for the whole signal. It's very interesting to note that the bidimensional projections of the reconstructed phase space (in the first few seconds this is the proper phase space because $d_{a} \simeq 2$ ) exhibit a behaviour close to a limit cycle, as one can see in Fig. 4a.

Figures $4 \mathrm{~b}-\mathrm{d}$ show the reconstructed phase space for the other three adjacent $3.5 \mathrm{~s}$ time windows. As we can see in particular in the last two, even if the dimension is lower than two, the scattering becomes present, partly obscuring the presence of a limit cycle. Finally, Fig. $4 \mathrm{e}$ and $\mathrm{f}$ show the phase space for time windows of 10 and $20 \mathrm{~s}$.

\section{Analytical effective dynamical model for the source}

The result of a dimension equal to two for the first few seconds of the signal could lead us to interpret our observation in terms of an harmonic oscillator, but the variability of the dimension throughout the time indicates the presence of a more complex dynamics (with a strange attractor and a dimension $2<d_{a}<3$ ) which starts its evolution over a limit cycle. A complete model of our signal requires a fluid-dynamic equa-

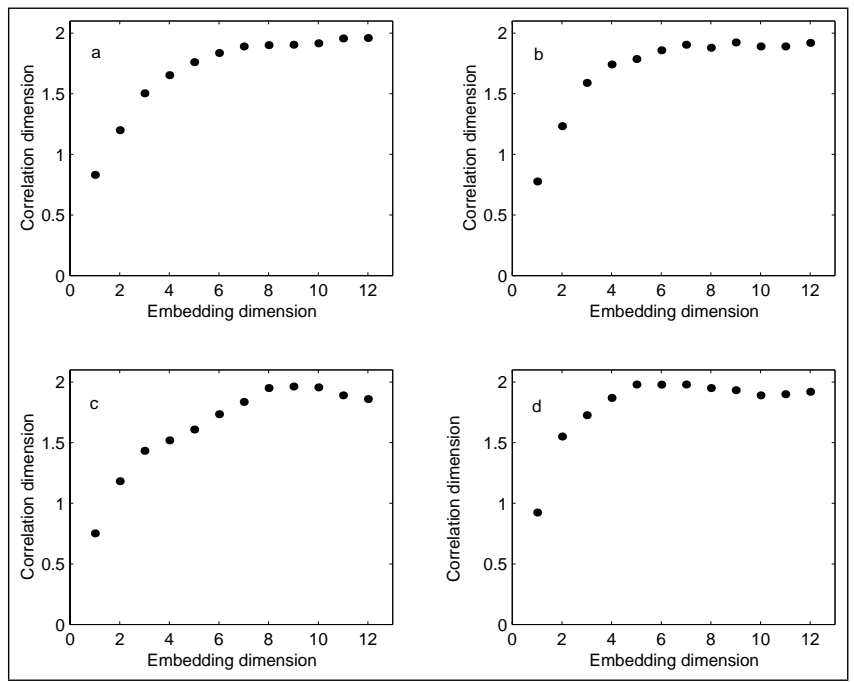

Fig. 2. Correlation dimension vs. embedding dimension as estimated by means of Grassberger and Procaccia method for the fourth adjacent $3.5 \mathrm{~s}$ time windows. As we can see, in all cases, $d_{a}$ tends toward two.

tion reproducing the signal and the phase space of Strombolian earthquakes. Here we limit our attention on an analogic model which should reproduce the characteristics of our signals. The idea is to choose, among various nonlinear oscillators, the one that best fits our data. The choice of a self-sustained oscillator has no particular reason except the one that it is the simplest nonlinear oscillator for which nonlinearity is clearly recognizable in the balancing of dissipation and loading mechanisms. An analogical example of self-sustained oscillations can be furnished by a valve oscillator with the oscillating circuit in the anode circuit and inductive feedback. Nonlinearity is produced by the mutual dependence between grid voltage and anodic current by inductive feedback. The system is described by the following adimensional couple of equations:

$\ddot{x}+h_{1} \dot{x}+h_{3} x=0$ for $x<b$,

$\ddot{x}-h_{2} \dot{x}+h_{3} x=0$ for $x>b$.

where $b$ is the first point of return of the limit cycle; $x$ is referred to a variable dynamically significant (current in the case of valve oscillator, ground displacement in our case); $h_{3}$ is a parameter connected to the characteristic frequency; $h_{1}$ is a dissipation parameter and $h_{2}$ is a loading parameter. For a detailed description of this equation, see Andronov et al. (1937). A discussion of the true physical meaning of $h_{1}$, $h_{2}, h_{3}$ is not possible at this stage, because our modelling is only analogic (see conclusion).

We fix $b$ observing the first return of the trajectory in the reconstructed phase space. To estimate the parameters $h_{1}$, $h_{2}$ and $h_{3}$, we construct a tridimensional matrix, whose elements generate, separately, a signal that can be compared to the true signal. We choose the best term in a sense of minimum square, i.e. we fix those parameters that generate a minimum root mean square deviation with respect to the original 


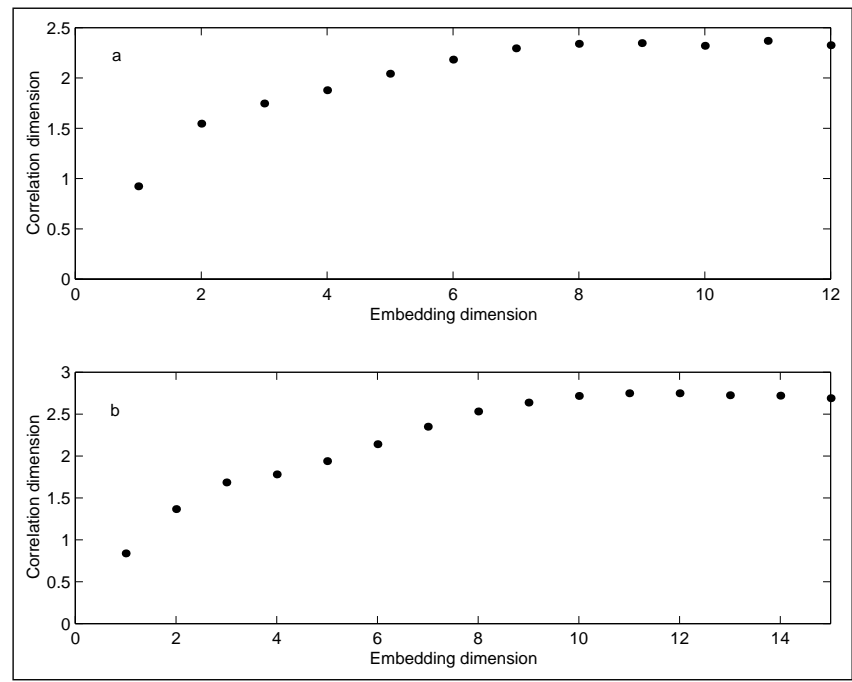

Fig. 3. Correlation dimension vs. embedding dimension as estimated by means of Grassberger and Procaccia method for two windows, respectively (a) $10 \mathrm{~s}$ and (b) $20 \mathrm{~s}$ long.

signal. The value of $\omega$ so obtained corresponds, within the statistical errors, to the first peak of the FFT of the original average earthquake, i.e. $1.1 \mathrm{~Hz}$. In Fig. 5 one can observe the original signal and the simulated one. The correlation coefficient between the two signals is $r=0.98$, the corresponding standard deviation is 0.04 . By changing instantaneously the value of $b$, it is possible to also simulate the next part of the signal, composed of the other 3-4 s. The entire signal can be obtained using Eq. (4) and introducing, where necessary, a short time instability by hand.

We have considered the explosion-quakes seismic signals of the Stromboli Volcano by studying their behaviour by means of standard non linear analysis of dynamical systems. The aim was to reconstruct an effective low-dimension dynamics characterizing the Stromboli source during this transient regime.

\section{Conclusions}

As a result, we have extracted phase space dimensions for the asymptotic time evolution. The dimension depends on the time length of the sample considered and ranges from 2 to 2.80 . This result could suggest that the signal is not stationary, but very simple statistical tests (multivariate analysis of the average value and of the variance) reveal that it is stationary at the first and second order. Such a peculiar result cannot be easily explained, but we suggest that the dynamics evolves in such a way that the whole phase space, in the first seconds, is not visible. In other words, the system should be in a stationary state which can be viewed only looking at the whole signal. The first seconds of the signals evolve on a stationary state but on a manifold of the phase space of lower dimensionality.
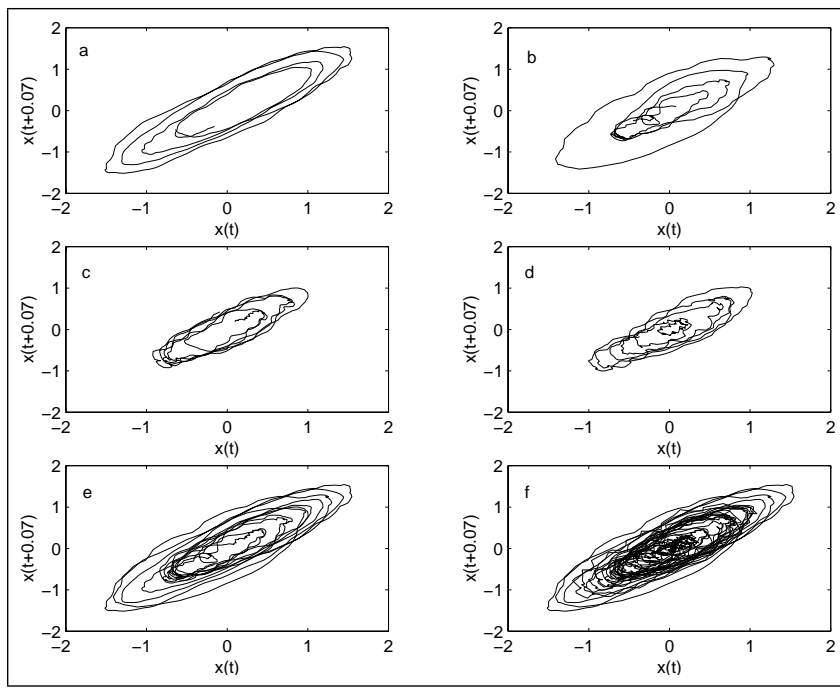

Fig. 4. Bidimensional projections of the reconstructed phase space with $\tau=0.07 \mathrm{~s}$. Figures (a), (b), (c), (d) show the phase space relative to $3.5 \mathrm{~s}$ time windows. Figure (e) shows the phase space of $10 \mathrm{~s}$ and Fig. (f) shows one relatively to the whole earthquake.

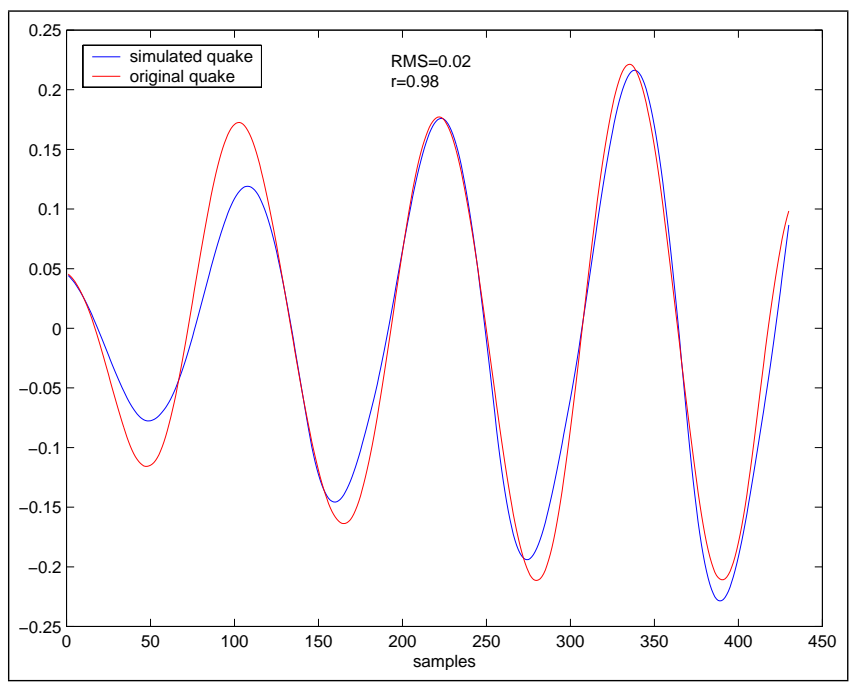

Fig. 5. Simulated earthquake and original one for the first $3.5 \mathrm{~s}$ (directly connected to the source); the coefficient of correlation is $r=0.98$ and $\mathrm{RMS}=0.02$.

We have focused our attention on this manifold because it is very simple to be reproduced. We have simulated these first seconds of the signal taking the general Eq. (4). This equation can give, fixing suitable parameters $h_{1}, h_{2}, h_{3}$ and $b$, all kinds of behaviour, i.e. harmonic, forced, damped and self-sustained oscillations. The best fit with the correlation equal to 0.98 and RMS equal to 0.04 singled out the selfsustained oscillator in the limit-cycle regime (see Fig. 5).

The estimated values of $h_{1}$ and $h_{2}$ give a numerical account of the balancing between dissipation and loading energy onto the magmatic system to generate the seismic signal. The characteristic oscillation frequency of this oscillator, $h_{3}$, coincides with the first peak of FFT of the true sig- 
nal. Note that our simulation is based on an analogic model which is able to simply reproduce the signal; this implies that a physical model should be based on more general equations which, with some simplification or averaging, should be transformed into Eq. (4). This and the reproduction of the whole phase space should be a matter of forthcoming papers.

\section{References}

Abarbanel, H. D. I.: Analysis of Observed Chaotic Data, SpringerVerlag, New York, Berlin, 1995.

Andronov, A. A., Vitt, A. A., and Khaikin, S. E.: Theory of oscillators, 1937, Republished Dover Publications, Inc., 1966.

Capuano, P. and Godano, C.: Source characterisation of low frequency events at Stromboli and Vulcano Islands (isole Eolie Italy), J. Seism, 3, 393-408, 1999.

Chouet, B. A. and Shaw, H. R.: Fractal properties of tremor and gas-piston events at Kilauea Volcano, Hawaii, J. Geophys. Res., 96, 1991.

Chouet, B. A., Saccorotti, G., Martini, M., Dawson, P., De Luca, G., Milana, G., and Scarpa, R.: Source and path effect in wavefield of tremor and explosions at Stromboli Volcano, Italy, J. Geophys. Res., 102, 15 129-15 150, 1997.

Del Pezzo, E., Godano, C., Gorini, A., and Martini, M.: Wave polarization and location of the source of the explosion quakes at Stromboli Volcano, IAVCEI Proceedings, in: Volcanology, (Eds) Gasparini, P., Scarpa, R., and Aki, K., Springer-Verlag, Berlin, 3, 279-295, 1992.

Fraser, A. M. and Swinney, H. L.: Independent coordinates for strange attractors from mutual information, Phys. Rev. A, 33,
1134-1139, 1986.

Grassberger, P. and Procaccia, I.: Measuring the strangeness of strange attractors, Physica D, 9, 189-208, 1983.

Grassberger, P., Hegger, R., Kanz, H., Schaffrath, C., and Schreiber, T.: On noise reduction methods for chaotic data, CHAOS, 3, 127, 1993.

Jaupart, C. and Vergniolle, S.: Laboratory models of Hawaiian and Strombolian eruptions, Nature, 331, 58-60, 1988.

Kennel, M. B., Brown, R., and Abarbanel, H. D. I.: Determining embedding dimension for phase space-reconstruction using a geometrical construction, Phys. Rev. A, 45, 3403-3411, 1992.

Kostelich, E. J. and Schreiber, T.: Noise reduction in chaotic time series data: A survey of common methods, Phys. Rev. E, 48, 1752-1763, 1993.

Mader, H. M., Zhang, Y., Phillips, J. C., Sparks, R. S., Sturtemant, B., and Stolper, E.: Experimental simulations of explosive degassing of magma, Nature, 372, 85-88, 1994.

Ripepe, M. and Gordeev, E.: Gas bubble dynamics model for shallow volcanic tremor at Stromboli, J. Geophys. Res., 104, 10639 $10654,1999$.

Schick, R. and Mueller, W.: Volcanic activity and eruption sequences at Stromboli during 1983-1984, Modelling of Volcanic Processes, (Eds) Chi-Yu King and Scarpa, Fr. Vieweg \& Son, Wiesbaden, 120-139, 1988.

Takens, F.: Detecting Strange Attractors in Turbolence, in Dynamical Systems and Turbolence Warwick, 1980, in Lectures Notes in Mathematics, 898, 366-381, Springer Berlin, 1981.

Vergniolle, S. and Brandeis, G.: Strombolian explosions 1. A large bubble breaking at the surface of a lava column as a source of sound, J. Geophys. Res., 101, 20 433-20 447, 1996. 